\title{
Epidemiology and Microbiology of Skin and Soft Tissue Infections: Preliminary Results of a National Registry
}

Silvano Esposito, Giuseppe De Simone, Angelo Pan, Paola Brambilla, Gianno Gattuso, Claudio Mastroianni, Blerta Kertusha, Carlo Contini, Lorenzo Massoli, Daniela Francisci, Giulia Priante, Marco Libanore, Roberto Bicocchi, Guglielmo Borgia, Alberto Enrico Maraolo, Pierluigi Brugnaro, Sandro Panese, Alessandra Calabresi, Giovanni Amendola, Francesca Savalli, Consuelo Geraci, Andrea Tedesco, Sara Fossati, Anna Carretta, Teresa Santantonio, Giovanni Cenderello, Maria Paola Crisalli, Elisabetta Schiaroli, Pierangelo Rovere, Giulia Masini, Roberto Ferretto, Antonio Cascio, Claudia Colomba, Claudia Gioè, Mario Tumbarello, Angela Raffaella Losito, Giuseppe Foti, Tullio Prestileo, Calogero Buscemi, Iaria Chiara, Carmelo lacobello, Sofia Sonia, Giulio Starnini, Anna Ialungo, Mauro Sapienza \& on behalf of the Italian Society of Infectious and Tropical Diseases

To cite this article: Silvano Esposito, Giuseppe De Simone, Angelo Pan, Paola Brambilla, Gianno Gattuso, Claudio Mastroianni, Blerta Kertusha, Carlo Contini, Lorenzo Massoli, Daniela Francisci, Giulia Priante, Marco Libanore, Roberto Bicocchi, Guglielmo Borgia, Alberto Enrico Maraolo, Pierluigi Brugnaro, Sandro Panese, Alessandra Calabresi, Giovanni Amendola, Francesca Savalli, Consuelo Geraci, Andrea Tedesco, Sara Fossati, Anna Carretta, Teresa Santantonio, Giovanni Cenderello, Maria Paola Crisalli, Elisabetta Schiaroli, Pierangelo Rovere, Giulia Masini, Roberto Ferretto, Antonio Cascio, Claudia Colomba, Claudia Gioè, Mario Tumbarello, Angela Raffaella Losito, Giuseppe Foti, Tullio Prestileo, Calogero Buscemi, Iaria Chiara, Carmelo lacobello, Sofia Sonia, Giulio Starnini, Anna lalungo, Mauro Sapienza \& on behalf of the Italian Society of Infectious and Tropical Diseases (2019) Epidemiology and Microbiology of Skin and Soft Tissue Infections: Preliminary Results of a National Registry, Journal of Chemotherapy, 31:1, 9-14, DOI: 10.1080/1120009X.2018.1536320

To link to this article: https://doi.org/10.1080/1120009X.2018.1536320

Published online: 03 Dec 2018.

Submit your article to this journal ¿

山 Article views: 60 
(n)

Full Terms \& Conditions of access and use can be found at

https://www.tandfonline.com/action/journallnformation?journalCode=yjoc20 


\section{Epidemiology and microbiology of skin and soft tissue infections: preliminary results of a National registry}

Silvano Esposito ${ }^{1}$, Giuseppe De Simone ${ }^{1}$, Angelo Pan ${ }^{2}$, Paola Brambilla², Gianno Gattuso ${ }^{3}$, Claudio Mastroianni ${ }^{4}$, Blerta Kertusha ${ }^{4}$, Carlo Contini ${ }^{5}$, Lorenzo Massoli ${ }^{5}$, Daniela Francisci ${ }^{6}$, Giulia Priante ${ }^{6}$, Marco Libanore ${ }^{7}$, Roberto Bicocchi ${ }^{7}$, Guglielmo Borgia ${ }^{8}$, Alberto Enrico Maraolo ${ }^{8}$, Pierluigi Brugnaro $^{9}$, Sandro Panese ${ }^{9}$, Alessandra Calabresi ${ }^{10}$, Giovanni Amendola ${ }^{10}$, Francesca Savalli ${ }^{11}$, Consuelo Geraci ${ }^{11}$, Andrea Tedesco ${ }^{12}$, Sara Fossati ${ }^{13}$, Anna Carretta ${ }^{14}$, Teresa Santantonio ${ }^{14}$, Giovanni Cenderello ${ }^{15}$, Maria Paola Crisalli $^{15}$, Elisabetta Schiaroli ${ }^{16}$, Pierangelo Rovere ${ }^{17}$, Giulia Masini ${ }^{17}$, Roberto Ferretto ${ }^{18}$, Antonio Cascio ${ }^{19}$, Claudia Colomba ${ }^{19}$, Claudia Giò̀ ${ }^{19}$, Mario Tumbarello ${ }^{20}$, Angela Raffaella Losito ${ }^{20}$, Giuseppe Foti ${ }^{21}$, Tullio Prestileo $^{22}$, Calogero Buscemi ${ }^{22}$, laria Chiara ${ }^{22}$, Carmelo lacobello ${ }^{23}$, Sofia Sonia $^{23}$, Giulio Starnini ${ }^{24}$, Anna lalungo ${ }^{24}$, Mauro Sapienza ${ }^{25}$, on behalf of the Italian Society of Infectious and Tropical Diseases

\footnotetext{
${ }^{1}$ Department of Infectious Diseases, University of Salerno, Salerno, Italy, ${ }^{2}$ Department of Infectious Diseases, Istituti Ospitalieri of Cremona, Cremona, Italy, ${ }^{3}$ Department of Infectious Diseases, Carlo Poma Hospital, Mantova, Italy, ${ }^{4}$ Department of Public Health and Infectious Diseases, Sapienza University, Latina, Italy, ${ }^{5}$ Department of Medical Sciences, Section of Infectious Diseases and Dermatology, University of Ferrara, Ferrara, Italy, "Infectious Diseases Clinic, University Hospital "S. Maria", University of Perugia, Perugia, Italy, ${ }^{7}$ Infectious Diseases Unit, Department of Infectious Diseases, S. Anna University Hospital, Ferrara, Italy, ${ }^{8}$ Department of Clinical Medicine and Surgery, Section of Infectious Diseases, University of Naples Federico II, Naples, Italy, 'Infectious Diseases Department, Ospedale Civile “SS. Giovanni e Paolo", Venice, Italy, ${ }^{10}$ Emergency Department, Hospital "Santi Antonio e Biagio e C. Arrigo", Alessandria, Italy, ${ }^{11}$ Infectious Diseases Unit, Hospital of Trapani, Trapani, Italy, ${ }^{12}$ Infectious Diseases Unit, Hospital Fracastoro San Bonifacio, Verona, Italy, ${ }^{13}$ Infectious Diseases Unit, University Hospital of Trieste, Trieste, Italy, ${ }^{14}$ Department of Infectious Diseases, University Hospital “Ospedali Riuniti” of Foggia, Foggia, Italy, ${ }^{15}$ Department of Infectious Diseases, Galliera Hospital, Genoa, Italy, ${ }^{16}$ Department of Medicine, Section of Infectious Diseases, University of Perugia, Perugia, Italy, ${ }^{17}$ Infectious Diseases Unit, Legnago Hospital, Verona, Italy, ${ }^{18}$ Infectious Diseases Unit, “Alto Vicentino” Santorso Hospital, Vicenza, Italy, ${ }^{19}$ Infectious Diseases Unit, Università degli Studi di Palermo, Palermo, Italy, ${ }^{20}$ Institute of Infectious Diseases, Foundation Policlinico Universitario A. Gemelli IRCCS, Cattolica del Sacro Cuore University, Rome, Italy, ${ }^{21}$ Infetious Diseases Unit, “Bianchi-Melacrino-Morelli” Hospital, Reggio Calabria, Italy, ${ }^{22}$ Infectious Diseases Unit, Arnas Civico Hospital, Palermo, Italy, ${ }^{23}$ Infectious Diseases Unit, Cannizzaro Hospital, Catania, Italy, ${ }^{24}$ Infectious Diseases Unit, Belcolle Hospital, Viterbo, Italy, ${ }^{25} \mathrm{UOC}$ Medicina Interna, Ospedale Umberto I, Enna, Italy
}

Skin and soft tissue infections (SSTIs) represent a wide range of clinical conditions characterized by a considerable variety of clinical presentations and severity. Their aetiology can also vary, with numerous possible causative pathogens. While other authors previously published analyses on several types of SSTI and on restricted types of patients, we conducted a large nationwide surveillance programme on behalf of the Italian Society of Infectious and Tropical Diseases to assess the clinical and microbiological characteristics of the 
whole SSTI spectrum, from mild to severe life-threatening infections, in both inpatients and outpatients. Twenty-five Infectious Diseases (ID) Centres throughout Italy collected prospectively data concerning both the clinical and microbiological diagnosis of patients affected by SSTIs via an electronic case report form. All the cases included in our database, independently from their severity, have been managed by ID specialists joining the study while SSTIs from other wards/clinics have been excluded from this analysis. Here, we report the preliminary results of our study, referring to a 12-month period (October 2016-September 2017). During this period, the study population included 254 adult patients and a total of 291 SSTI diagnoses were posed, with 36 patients presenting more than one SSTIs. The type of infection diagnosed, the aetiological microorganisms involved and some notes on their antimicrobial susceptibilities were collected and are reported herein. The enrichment of our registry is ongoing, but these preliminary results suggest that further analysis could soon provide useful information to better understand the national epidemiologic data and the current clinical management of SSTIs in Italy.

Keywords: Epidemiology, microbiology, skin and soft tissue infections, registry

\section{Introduction}

Skin and soft tissue infections (SSTIs) are some of the most commonly occurring bacterial infections, with a wide range of possible aetiological pathogens and a considerable variety of clinical presentations and severity, from mild to severe life-threatening infections. The characteristic variability of such infections makes it difficult to classify them and define their prevalence with certainty. Moreover, many cases of common and relatively less severe SSTIs (such as cellulitis and small subcutaneous abscesses) are not routinely cultured. Therefore, in most cases the precise microbiological causes of these infections remain unknown. ${ }^{1}$ Given the epidemiological importance assumed by SSTIs during the past two decades, with a marked rise in incidence worldwide, ${ }^{2,3}$ and the paucity of broad population-based studies on SSTIs we conducted a nationwide surveillance programme to assess the clinical and microbiological characteristics of the whole spectrum of SSTIs (ranging from simple folliculitis to severe necrotizing fasciitis). Here, we report the preliminary results of our study and compare our findings with those reported in the literature.

\section{Patients and methods}

Twenty-five Infectious Diseases Centres throughout Italy (eight in the Northern, eight in Middle and nine in Southern Italy) collected prospectively data concerning the diagnosis and management of both inpatients and outpatients affected by SSTI managed by ID specialists, via an electronic case report form. For each clinical case entered into the database, the following information were collected: patients' demographic data; the setting where they come from (community, emergency room, hospital or long-term care facilities); type of SSTI diagnosed; site and size of the lesions; co-morbidities; results of microbiological tests, including notes on the patterns of antibiotic susceptibilities of the microorganisms isolated; type and results of previous treatment; type and results of current antibiotic treatment.

The study was approved by the local Ethical Committee (Comitato Etico Campania Sud approval 84/2016) for the principal investigator. The study was approved as well by the single Ethical Committees for each participating centre.

The study is still ongoing and only some preliminary results are presented herein. The registry is constantly expanding and the study population increasing; in the present study, we limited our observation to a restricted period (from October 2016 to September 2017) and we only focussed on some of the information available in the database: we analysed the preliminary data on the type of infection and the aetiological agents, while a more complete far-reaching analysis of the whole registry will soon be undertaken.

\section{Results}

During the 12 months period, the ID specialists of the 25 ID centres involved in the study, enrolled 254 adult patients diagnosed with SSTI: 171 males $(67.3 \%)$ and 83 females $(32.7 \%)$ with a male/female ratio of 2.06. The age range of the population was $18-98$ years, with an average age of 60.04 years and a median age of 59 years. Indeed, the highest incidence rate of SSTI was observed in the range 50-69 years (104 cases, $41 \%$ ), often presenting a high degree of co-morbidity, the most common being diabetes mellitus (30.7\% of patients) and cardiovascular diseases (30\% of patients). The majority of SSTIs $(55.9 \%)$ were diagnosed in patients coming from community rather than health care facilities.

In the 254 patients, a total of 291 diagnoses of SSTIs were made with 36 patients presenting more than one SSTI. The most common diagnoses were: cellulitis $(29 \%)$, erysipelas $(14.1 \%)$, surgical site infections (10.3\%) and diabetic foot infection $(8.6 \%)$ as shown in Table 1. No relevant differences of SSTI rates among the centres joining the study 
Table 1. SSTI diagnosis posed in the study population.

\begin{tabular}{lcc}
\hline SSTI & \multicolumn{2}{c}{$\begin{array}{c}\text { Number of cases out of } \\
\text { total SSTIs (\%) }\end{array}$} \\
\hline Cellulitis & 84 & $(29)$ \\
Erysipelas & 41 & $(14.1)$ \\
Surgical site infection & 30 & $(10.3)$ \\
Diabetic foot infection & 25 & $(8.6)$ \\
Abscess & 23 & $(8)$ \\
Venous ulcer & 21 & $(7.2)$ \\
Phlegmon & 19 & $(6.5)$ \\
Wound infection & 9 & $(3.1)$ \\
Pressure ulcer & 6 & $(2.1)$ \\
Impetigo & 4 & $(1.4)$ \\
Necrotizing fasciitis & 3 & $(1)$ \\
Hidradenitis & 3 & $(1)$ \\
Myonecrosis & 2 & $(0.7)$ \\
Folliculitis & 1 & $(0.3)$ \\
Forunculosis & 1 & $(0.3)$ \\
Clostridial myonecrosis & 1 & $(0.3)$ \\
Bite wound infection & 1 & $(0.3)$ \\
Other infection & 17 & $(5.8)$ \\
Total & 291 & \\
\hline
\end{tabular}

throughout the country has been found. The majority of the lesions were located in the lower limbs $(67 \%)$, followed by higher limbs $(10.2 \%)$ and trunk (5.5\%). Sixty-eight cases out of the total 291 SSTIs diagnosed in our study met the criteria for Acute Bacterial Skin and Skin-Structure Infection (ABSSSI) definition including the size of lesion. Most of them were cellulitis and erysipelas (47 and $39 \%$, respectively).

Microbiological assessment of the lesions was performed through the culture of several specimens depending on the particular type of infection: superficial swabs; deep tissue biopsy and blood. For 78 patients $(30.7 \%)$, most of whom were affected by cellulitis and erysipelas, no microbiological exam was requested. For the remaining 176 patients, a total of 187 microbiological exams were implemented (Table 2). Of the 187 exams, 45 (24.1\%) did not yield bacterial growth (cellulitis, erysipelas and surgical wound reporting the highest failure rates for microbial assessment). Of the 142 pathogen-positive cultures, multiple micro-organisms were identified in 50 cases $(35.2 \%)$, surgical wounds and diabetic foot infections accounting for almost half of such cases (10 and 8, respectively), while a single micro-organism was detected in the remaining 92 cultures $(64.8 \%)$. In Table 3, we present the number of overall isolates (194 bacterial species) yielded by the 142 positive cultures and we further distinguish the microbiological results of monomicrobial and polymicrobial isolates. The most common bacterial species found as causative agents of overall SSTIs were represented by Grampositive bacteria [108 (56\%)] while Gram-negatives accounted for $64(33 \%)$ as shown in Table 3 . Staphylococcus aureus were detected in 74 cases
(38.2\%), being the leading cause of such infections, with a methicillin-resistance rate of $57 \%$ (Table 4). The second and the third most common aetiological pathogens in our database were represented by Pseudomonas spp. and Enterococcus faecalis, with $32(16.5 \%)$ and $13(6.7 \%)$ cases, respectively.

\section{Discussion}

Skin and soft tissue infections have always been among the most common bacterial infections but their incidence has worryingly increased worldwide during last two decades, assuming the proportion of a global public health threat. ${ }^{2-6}$ One of the most recent large-scale studies conducted on the HealthCore Integrated Research Database demonstrated that the SSTI incidence is about 48.46 cases/1000 population year. ${ }^{2}$ Moreover, the emergence of multi-drug resistant microorganisms (first of all the notorious MRSA) and their spread, not only among health care facilities, but also in community settings makes this phenomenon even more alarming and the management of such infections more challenging due to the reduced efficacy of available antimicrobial armamentarium. ${ }^{7-11}$ Therefore, knowledge of SSTI epidemiology and the current economic healthcare burden is crucial to combat these infections but the range of SSTIs is so heterogeneous and complex that, although several classifications have been drawn up over the years on the basis of several variables (aetiology, localization, clinical severity...), no classification has received universal consensus. ${ }^{1,12,13}$ Several authors have already published investigations on clinical presentation and microbiological assessment of SSTIs but they often limited their study population to restricted types of patients such as hospitalized patients, for example, ${ }^{14,15}$ or to restricted diagnosis, such as complicated SSTIs. ${ }^{14-16}$ Here, we report the preliminary results of a nationwide surveillance programme including all categories of SSTIs and patients deserving consultation with an infectious diseases specialist either in the clinic, or in the ward or in the emergency room (from the common and uncomplicated folliculitis in otherwise healthy patients to the severe necrotizing infection in critically ill patients), through the use of an electronic registry designed and tailored to collect a wide range of information. Although many SSTIs are managed by other specialists than ID specialists in surgical and clinical wards, we did not include in our analysis the cases from other wards than ID units and we focussed our observation on all the cases, independently from their severity, that came to the attention of the ID specialists joining the study. 
The demographic characteristics of our study population were similar to those of other studies previously published, chiefly consisting of older patients $(54.3 \%>60$ years old $)$ with a male/female ratio $>1$, although our ratio (2.06) was considerably higher than that found by Gibbons $(1,38)^{16}$ and by Garau (1.36). ${ }^{14}$ Diabetes mellitus proved to be the most frequent comorbidity among our patients $(30.7 \%$ of total patients suffering), which means that diabetes mellitus occurs approximately five times more frequently in SSTI patients than in the general population, a similar percentage being found by Garau $\left(33,9 \% ;{ }^{14}\right)$. The above data strongly suggest that diabetics have an increased risk of SSTIs, maybe due to impaired immunologic response to pathogens. ${ }^{7,17}$ Moreover, although there is no universal agreement on how far this increased risk is directly related to diabetes, recent large-scale investigations have demonstrated that not only are diabetics inclined to develop SSTIs but they also have a five times higher risk of SSTIassociated complications compared to patients without diabetes. ${ }^{18,19}$ It is hardly surprising that in our registry, diabetic foot infection (DFI) is the

Table 2. Microbiological tests performed for diagnosis.

\begin{tabular}{lc}
\hline Study population & $\mathbf{2 5 4}$ \\
\hline Patients with no microbiological & 78 \\
$\quad$ exams performed & 176 \\
Patients with at least one & \\
$\quad$ microbiological exam performed & Number of cases \\
Microbiological exams performed & 74 \\
Superficial swab & 51 \\
Blood culture & 38 \\
Deep tissue & 17 \\
Biopsy & 7 \\
Other & 187 \\
Total & \\
\hline
\end{tabular}

most frequently occurring infection in diabetics (27.3\% of SSTIs diagnosed in diabetic patients) but it is noteworthy that also other types of infections are often diagnosed: cellulitis $(23.9 \%)$, surgical wound infection $(11.4 \%)$, abscess $(10.3 \%)$ and erysipelas $(9.1 \%)$. Therefore, diabetics need particular care to diagnose early on and prevent any SSTIs (not only DFI) and their complications.

A total of 291 diagnoses of SSTIs were posed in our registry, the most common being cellulitis, representing $29 \%$ of overall SSTIs, followed by erysipelas $(14.1 \%)$, surgical site infections $(10.3 \%)$, DFI $(8.6 \%)$ and abscess $(8 \%)$. Comparing our data with those reported elsewhere, the percentages of cellulitis and abscesses appear to be lower, but it should be noted that in many studies abscesses and cellulitis are considered in one category (thus the group of cellulitis/abscess easily reaches higher percentages, from $50 \%{ }^{15}$ to $63 \%^{7}$ ). Furthermore, while in our analysis $14.1 \%$ of the overall SSTIs were diagnosed as erysipelas, other authors considered erysipelas in the same category as cellulitis/abscess with no further distinctions. Thus, adding the percentages of cellulitis, abscess and erysipelas diagnosed in our registry we obtain a $51 \%$ that is quite similar to previously published data. Also, the rates of DFI (8.6\%), surgical wound (10.3\%) and trophic ulcer $(7.2 \%)$ are much closer to data published by Garau et al. ${ }^{9}$ Yet it is significant that impetigo, forunculosis and folliculitis, representing less than $2 \%$

Table 4. Methicillin-resistance rate among $S$. aureus isolated.

\begin{tabular}{lc}
\hline S. aureus & 74 isolates (\%) \\
\hline MRSA & $42(57)$ \\
MSSA & $32(43)$
\end{tabular}

Table 3. Bacterial species isolated from monomicrobial and polymicrobial cultures.

\begin{tabular}{|c|c|c|c|c|c|c|}
\hline \multirow{2}{*}{$\begin{array}{l}\text { Bacterial species } \\
\text { Gram positives }\end{array}$} & \multicolumn{2}{|c|}{$\begin{array}{l}\text { Number (\%) of colonies } \\
\text { from the overall cultures }\end{array}$} & \multicolumn{2}{|c|}{$\begin{array}{l}\text { Number }(\%) \text { of colonies from } \\
\text { the monomicrobial cultures }\end{array}$} & \multicolumn{2}{|c|}{$\begin{array}{l}\text { Number (\%) of colonies from } \\
\text { the polymicrobial cultures }\end{array}$} \\
\hline & 108 & (56) & 65 & $(70.7)$ & 43 & $(42.2)$ \\
\hline S. aureus & 74 & (38.2) & 51 & (55.4) & 23 & (22.6) \\
\hline E. faecalis & 13 & $(6.7)$ & 3 & (3.3) & 10 & $(9.8)$ \\
\hline Coag.neg. Staph. & 9 & (4.6) & 6 & (6.5) & 3 & (2.9) \\
\hline S. agalactiae & 7 & (3.6) & 5 & (5.4) & 2 & (1.9) \\
\hline E. faecium & 4 & (2.2) & 0 & (0) & 4 & (3.9) \\
\hline S. pyogenes & 1 & (0.5) & 0 & (0) & 1 & (0.9) \\
\hline Gram negatives & 64 & (33) & 22 & (24) & 42 & (41.2) \\
\hline $\begin{array}{l}\text { Pseudomonas } \\
\text { spp. }\end{array}$ & 32 & $(16.5)$ & 13 & $(14.1)$ & 19 & (18.6) \\
\hline E. coli & 13 & (6.7) & 3 & (3.3) & 10 & (9.8) \\
\hline $\begin{array}{l}\text { Acinetobacter } \\
\text { spp. }\end{array}$ & 6 & (3.1) & 2 & (2.2) & 4 & (3.9) \\
\hline Proteus spp. & 5 & (2.6) & 2 & $(2.2)$ & 3 & (2.9) \\
\hline Enterobacter spp. & 5 & (2.6) & 2 & (2.2) & 3 & (2.9) \\
\hline Klebsiella spp. & 3 & (1.5) & 0 & (0) & 3 & (2.9) \\
\hline Other species & 22 & (11) & 5 & (5.3) & 17 & (16.6) \\
\hline Total & 194 & & $92 / 194$ & & $102 / 19$ & \\
\hline
\end{tabular}


of the SSTIs diagnosed in our study, are described with a much higher proportion of diagnoses in other studies (12\% impetigo and 17\% forunculosis for Casey et $\mathrm{al}^{20}{ }^{20}$. All these differences present in the literature in the rates of various SSTIs are due to several biases, first of all the selection of patients (as the frequency of some infections markedly differs between inpatients and outpatients) but also the selection of the type of SSTI included in the study. In our registry, we included a large cohort of different patients and all types of SSTIs, from mild infections not needing drugs, to severe, necrotic and complicated infections with a view to representing accurately the heterogeneous array of SSTIs.

In our database, the microbiological exams most often required to identify the aetiological pathogens of the SSTI are the culture of superficial swabs, deep tissue biopsy and blood. However, the reason for seeking aetiological exams depends on the type of infection. Thus, microbiological tests are not always needed to pose the clinical diagnosis of some less severe SSTIs or when the aetiology is supposed to be known. ${ }^{21,22}$ Indeed, in $30.7 \%$ of the cases in this study, the patients did not undergo a microbiological test as it was considered unnecessary (most often for cellulitis, erysipelas and small subcutaneous abscess) and antimicrobial treatment was started without a microbial diagnosis. This applies to a new clinical entity, recently defined by the US Food and Drug Administration (FDA): acute bacterial skin and skin-structure infection (ABSSSI). ABSSSI is defined as a bacterial infection of the skin with a lesion size area of $\geq 75 \mathrm{~cm}^{2}$ (lesion size measured by the area of redness, oedema or induration), including cellulitis/erysipelas, wound infections and major cutaneous abscesses. ${ }^{23-25}$ A total of $47 \%$ of the cellulitis and $39 \%$ of the erysipelas in our registry have met the criteria for ABSSSI definition. Such infections, although presenting different clinical entities, share the same aetiology (Staphylococcus aureus and/or Streptococcus pyogenes). Hence, the appropriate antimicrobial therapy can easily be administered without delay. ${ }^{12}$ Yet, for most of the other SSTIs, the identification of the causative pathogen and the assessment of its antimicrobial susceptibility profile can be crucial to select the most effective antibiotic therapy, although there is no agreement on the most reliable tool for SSTI microbiological diagnosis. As expected, in our registry, the superficial swab is the most commonly adopted method for the collection of clinical samples (74 of the 187 microbiological tests; $39.6 \%$ ) while the culture of deep tissue biopsy was performed only in 55 cases (55/187, 29.4\%). Though many authors still debate about the rate of concordance between these two methods, ${ }^{26-28}$ most of the guidelines indicate that the culture of deep tissue sampling (collected by curettage and biopsy or aspiration of infected secretions) provides the most useful information on the causative organisms often lying in deeper layers, while the cultures of superficial swabs are easily contaminated by harmless bacteria belonging to indigenous microbial flora. ${ }^{26,29}$ Of the overall 187 microbiological exams performed in our study, $45(24.1 \%)$ failed to detect any microorganisms; blood culture reporting the highest negative rate (no bacterial growth in 25 out of 52 blood cultures, $49 \%$ ): indeed bacteraemia is uncommon in mild SSTI and guidelines recommend blood cultures only for severe SSTIs requiring hospitaliszation. ${ }^{22,30}$ Upon analysing the 142 positive cultures, we noted that multiple pathogens were isolated in 50 cases $(35.2 \%)$, especially from surgical wound and diabetic foot infections; while a single microorganism had been detected in the remaining 92 cultures $(64.8 \%)$. In more than half of the SSTIs $(56 \%)$, Gram-positive bacteria were detected as the aetiological pathogen. These data are very similar to the findings of Garau et al. $(57 \%)^{14}$ but lower than that reported by Ray et al. $(87 \%)^{7}$ and Tiwari et al. $(70.5 \%) .{ }^{31} S$. aureus represents by far the most common bacterial species detected also in the other studies cited above but we found a higher proportion of methicillin-resistance among the $S$. aureus isolates $(56.2 \%$ of the overall $S$. aureus isolated were MRSA) compared to that reported by these authors $\left(46 \%{ }^{7}\right.$ and $\left.27.6 \%{ }^{14}\right)$. The percentage of Gram-negatives among the causative pathogens was $33 \%$, very similar to that found by Garau et al. (35\%) but differently from their results, in which Enterobacteriaceae accounted for $25 \%$ of all the causative agents, we found that $P$. aeruginosa was the most common (16.5\%), while Enterobacteriaceae were isolated only in $10.8 \%$ of cases. Enterococcus faecalis (6.7\%), Acinetobacter spp. (3.1\%), Enterobacter spp. (2.6\%), Enterococcus faecium (2.2\%), coag. neg Staphylococci $(4.6 \%)$ and $S$. pyogenes $(0.5 \%)$ were less frequently isolated as the role of these pathogens seems to be secondary, as was found in other studies.

In conclusion, these preliminary results of our nationwide surveillance programme show that the registry, during the first 12 months period, has collected a considerable amount of data, partly overlapping with those of previous large-scale studies but slightly diverging from them as regards the prevalence of some specific categories of infections, or the different share of some aetiological agents. Therefore, the continuous enrichment of this registry until the end of the study period (2 years), 
could soon provide us useful information to represent a detailed picture of the national epidemiologic data and the current clinical management of SSTIs in Italy, both for their diagnostic and therapeutic approach.

\section{Disclosure statement}

No potential conflict of interest was reported by the authors.

\section{References}

1 Esposito S, Bassetti M, Concia E, De Simone G, De Rosa FG, Grossi P, et al. Diagnosis and management of skin and soft-tissue infections (SSTI). A literature review and consensus statement: an update. J Chemother. 2017;29(4):197-214.

2 Miller LG, Eisenberg DF, Liu H, Chang CL, Wang Y, Luthra $\mathrm{R}$, et al. Incidence of skin and soft tissue infections in ambulatory and inpatient settings, 2005-2010. BMC Infect Dis. 2015; $15: 362$.

3 Esposito S, Noviello S, Leone S. Epidemiology and microbiology of skin and soft tissue infections. Curr Opin Infect Dis. 2016;29(2):109-15.

4 Edelsberg J, Taneja C, Zervos M, Haque N, Moore C, Reyes $\mathrm{K}$, et al. Trends in US hospital admissions for skin and soft tissue infections. Emerging Infect. Dis. 2009;15(9):1516-18.

5 Marra F, Patrick DM, Chong M, McKay R, Hoang L, Bowie WR. Population-based study of the increased incidence of skin and soft tissue infections and associated antimicrobial use. Antimicrob Agents Chemother. 2012;56(12):6243-9.

6 Pallin DJ, Espinola JA, Leung DY, Hooper DC, Camargo Jr CA. Epidemiology of dermatitis and skin infections in United States physicians' offices, 1993-2005. Clin Infect Dis. 2009; 49(6):901-7.

7 Ray GT, Suaya JA, Baxter R. Incidence, microbiology, and patient characteristics of skin and soft-tissue infections in a U.S. population: a retrospective population-based study. BMC Infect Dis. 2013;13:252.

8 Esposito S, De Simone G. Update on the main MDR pathogens: prevalence and treatment options. Infez Med. 2017;25(4): 301-10.

9 Shahini Shams Abadi M, Nikokar I, Hoseini Alfatemi SM, Malekzadegan Y, Azizi A, Sedigh Ebrahim-Saraie H. Epidemiology of Panton-Valentine Leukocidin harbouring Staphylococcus aureus in cutaneous infections from Iran: a systematic review and meta-analysis. Infez Med. 2017;25(3): 217-23.

10 Leone S, Noviello S, Boccia G, De Caro F, Esposito S. Methicillin-resistant Staphylococcus aureus infections: role of daptomycin/ $\beta$-lactams combination. Infez Med. 2015;23(2): 99-104

11 Cascone C, Santagati M, Noviello S, Iannelli F, Esposito S, Pozzi G, et al. S. Macrolide-resistance genes in clinical isolates of Streptococcus pyogenes. Microb Drug Resist. 2002;8(2): 129-32.

12 Esposito S, Noviello S, De Caro F, Boccia G. New insights into classification, epidemiology and microbiology of SSTIs, including diabetic foot infections. Infez Med. 2018;26(1):3-14.

13 Esposito S, Bassetti M, Borre' S, Bouza E, Dryden M, Fantoni $\mathrm{M}$, et al. Diagnosis and management of skin and soft-tissue infections (SSTI): a literature review and consensus statement on behalf of the Italian Society of Infectious Diseases and International Society of Chemotherapy. J Chemother. 2011; 23(5):251-62

14 Garau J, Blasi F, Medina J, McBride K, Ostermann H; REACH study group. Early response to antibiotic treatment in European patients hospitalized with complicated skin and soft tissue infections: analysis of the REACH study. BMC Infect Dis. 2015;15:78.

15 Shen HN, Lu CL. Skin and soft tissue infections in hospitalized and critically ill patients: a nationwide population-based study. BMC Infect Dis. 2010;10:151.

16 Gibbons JA, Smith HL, Kumar SC, Duggins KJ, Bushman AM, Danielson JM, et al. Antimicrobial stewardship in the treatment of skin and soft tissue infections. Am J Infect Control 2017;45(11):1203-7.

17 Leone S, Pascale R, Vitale M, Esposito S. Epidemiology of diabetic foot. Infez Med. 2012;20(1):8-13.

18 Suaya JA, Eisenberg DF, Fang C, Miller LG. Skin and soft tissue infections and associated complications among commercially insured patients aged 0-64 years with and without diabetes in the U.S. PLoS One 2013;8(4):e60057.

19 Muller LM, Gorter KJ, Hak E, Goudzwaard WL, Schellevis FG, Hoepelman AI, et al. Increased risk of common infections in patients with type 1 and type 2 diabetes mellitus. Clin Infect Dis. 2005;41(3):281-8.

20 Casey JA, Cosgrove SE, Stewart WF, Pollak J, Schwartz BS. A population-based study of the epidemiology and clinical features of methicillin-resistant Staphylococcus aureus infection in Pennsylvania, 2001-2010. Epidemiol Infect. 2013;141(6): 1166-79.

21 Stevens DL, Bisno AL, Chambers HF, Dellinger EP, Goldstein EJ, Gorbach SL, et al. Practice guidelines for the diagnosis and management of skin and soft tissue infections: 2014 update by the Infectious Diseases Society of America. Clin Infect Dis. 2014;59(2):e10-52.

22 Esposito S, Bassetti M, Bonnet E, Bouza E, Chan M, De Simone $\mathrm{G}$, et al. Hot topics in the diagnosis and management of skin and soft-tissue infections. Int J Antimicrob Agents 2016; 48(1):19-26.

23 US Food and Drug Administration, Center for Drug Evaluation and Research. Guidance for Industry. Acute Bacterial Skin and Skin Structure Infections: Developing Drugs for Treatment. Draft Guidance. 2010. Clinical/antimicrobial revision 1. Retrieved from http://www.fda.gov/downloads/ Drugs/ .../Guidances/ ucm071185.pdf

24 Esposito S., Noviello S., Leone S. Dalbavancin for the treatment of acute bacterial skin and skin structure infections. Infez Med. 2015;23(4):313-17.

25 Russo A, Concia E, Cristini F, De Rosa FG, Esposito S, Menichetti F, et al. Current and future trends in antibiotic therapy of acute bacterial skin and skin-structure infections. Clin Microbiol Infect. 2016;22(2):S27-36.

26 Esposito S, De Simone G, Gioia R, Noviello S, Pagliara D, Campitiello N, et al. Deep tissue biopsy vs. superficial swab culture, including microbial loading determination, in the microbiological assessment of Skin and Soft Tissue Infections (SSTIs). J Chemother. 2017;29(3):154-8

27 Mutluoglu M, Uzun G, Turhan V, Gorenek L, Ay H, Lipsky BA. How reliable are cultures of specimens from superficial swabs compared with those of deep tissue in patients with diabetic foot ulcers? J Diabetes Complications 2012;26(3):225-9.

28 Nelson A, Wright-Hughes A, Backhouse MR, Lipsky BA, Nixon J, Bhogal MS, et al. CODIFI (Concordance in Diabetic Foot Ulcer Infection): a cross-sectional study of wound swab versus tissue sampling in infected diabetic foot ulcers in England. BMJ Open 2018;8(1):e019437.

29 Lipsky BA, International consensus group on diagnosing and treating the infected diabetic foot. A report from the international consensus on diagnosing and treating the infected diabetic foot. Diabetes Metab Res Rev. 2004;20(S1):S68-S77.

30 Saeed K, Esposito S, Gould I, Ascione T, Bassetti M, Bonnet $\mathrm{E}$, et al. Hot topics in necrotising skin and soft tissue infections. Int J Antimicrob Agents 2018;52(1):1-10.

31 Tiwari AK, Lal R. Study to evaluate the role of severity stratification of skin and soft tissue infections (SSTIs) in formulating treatment strategies and predicting poor prognostic factors. Int J Surg. 2014;12(2):125-33. 